\title{
Aiming for a shorter time to diagnosis: pediatric eosinophilic esophagitis in British Columbia
}

Jocelyn Jia ${ }^{1,2^{*}}$, Edmond S. Chan ${ }^{2,3}$, Vishal Avinashi ${ }^{2,4}$, Elaine Hsu ${ }^{2,3}$, Hin Hin Ko ${ }^{5,6}$ and Lianne Soller 2,3

\begin{abstract}
Longer time to diagnosis for patients with eosinophilic esophagitis can lead to adverse patient outcomes, but the length of diagnostic delay has not been quantified for patients with eosinophilic esophagitis in Canada. Our study defines the time to diagnosis (TTD) for pediatric patients with eosinophilic esophagitis in British Columbia and identifies factors that predict increased time to diagnosis. The median TTD was 21 months $(1.75$ years; IQR $=7,45)$ with a median age at EoE diagnosis of 105 months ( 8.75 years; IQR $=44,156)$. Caucasians experienced significantly longer TTD compared to other ethnicities ( 24 months (IQR $=7,52)$ and 12 months (IQR $=4.5,23)$ respectively, $p=0.008)$. Caucasian ethnicity $(p=0.037)$ and older age at the time of diagnosis $(p=0.006)$ predicted increased TTD. Our model explained $7.9 \%$ (Adjusted $R^{2}=0.079$ ) of the total variance for our cohort.
\end{abstract}

Keywords: Eosinophilic esophagitis, Pediatric, Diagnosis, Delay

\section{Main text}

Eosinophilic esophagitis (EoE) is a chronic allergic condition increasing in incidence worldwide, though epidemiological data describing EoE in Canada are lacking [1]. Symptoms of EoE are often nonspecific and can differ by age [1-3], making timely and accurate diagnosis a challenge. No studies have explored time to diagnosis (TTD) for EoE in Canada. Our goals were to describe TTD and factors associated with TTD for children enrolled in the EoE Registry at British Columbia Children's Hospital (BCCH).

This study was approved by the University of British Columbia Children's and Women's Research Ethics Board. $\mathrm{BCCH}$ is the sole pediatric tertiary care facility in British Columbia, and is responsible for most pediatric EoE patients in the province. Patients seen in the multidisciplinary EoE clinic-comprised of one pediatric allergist, one pediatric gastroenterologist, and

\footnotetext{
*Correspondence: jocelyn.jia@mail.utoronto.ca

1 Faculty of Medicine, University of Toronto, Toronto, ON, Canada

Full list of author information is available at the end of the article
}

one dietician-at $\mathrm{BCCH}$ were eligible for the current study if they were: $(1)<18$ years old, (2) resided in BC, (3) had EoE symptoms preceding a biopsy-proven EoE diagnosis $(\geq 1$ biopsy showing $\geq 15$ eosinophils/highpowered field), and (4) consented to participate in the EoE Registry. Data have been entered prospectively into the Registry since 2012 and stored in Research Electronic Data Capture (REDCap), a secure web-based data management software [4].

A cross-sectional analysis using TTD (time between EoE symptom onset and diagnostic endoscopy) as the dependent variable was performed. Independent variables were sex, location (close to tertiary hospital: Vancouver Coastal and Fraser Health Authorities; far from tertiary hospital: Vancouver Island, Interior, and Northern Health Authorities), ethnicity (Caucasian or Other), past history of gastrointestinal (GI) issues (any self-reported GI conditions), past history of allergic conditions (asthma, atopic dermatitis, food allergy, allergic rhinitis, pollen-food syndrome, and/ or environmental allergy), family history (first-degree relatives) of GI issues, family history of EoE, and 
family history of allergic conditions. This information was self-reported by patients or their parent/ guardian. Kolmogorov-Smirnov, Mann-Whitney U tests, and Kruskal-Wallis $\mathrm{H}$ tests were performed. A multivariable linear regression was performed using TTD as the dependent variable and the following independent variables: sex, location, ethnicity, past history of GI issues, past history of allergic conditions, family history of GI issues, family history of EoE, family history of allergic conditions, and age at diagnosis. SPSS Statistical Package (IBM Corp. Released 2019. IBM SPSS Statistics for Windows, Version 26.0. Armonk, NY: IBM Corp.) was used. Significance was set at $p<0.05$.

Between July 10th, 2012 and June 8th, 2018, 169 patients were enrolled in the EoE Registry. Patients with missing data $(n=50)$ or who did not meet the inclusion criteria $(\mathrm{n}=4)$ were excluded, leaving 115 patients for analysis. The study population was $80.0 \%$ (92/115) male and $73.9 \%(85 / 115)$ Caucasian with the rest being predominantly South Asian (Table 1). The median age at symptom onset was 72 months (6 years; $\mathrm{IQR}=13,122)$, the median age at EoE diagnosis was 105 months (8.75 years; IQR $=44,156)$, and the median TTD was 21 months ( 1.75 years; IQR $=7,45$; Table 1 ).

In our cross-sectional analysis, Caucasians experienced significantly longer TTD compared to other ethnicities ( 24 months (IQR $=7,52)$ and 12 months (IQR $=4.5,23)$ respectively, $p=0.008$, Table 2). No other variables were significant. Multivariate linear regression determined that Caucasian ethnicity $(p=0.037)$ and older age at the time of diagnosis $(p=0.006)$ predicted increased TTD. No other variables were significant. Our model

\section{Table 1 Demographic characteristics}

\begin{tabular}{lc}
\hline Characteristic & Total $(\mathbf{n = 1 1 5 )}$ \\
\hline Age at symptom onset in months, median (IQR) & $72(13,122)$ \\
Age at diagnosis in months, median (IQR) & $105(44,156)$ \\
TTD in months, median (IQR) & $21(7,45)$ \\
Male & $92(80 \%)$ \\
Close to tertiary hospital (Vancouver Fraser and & $73(63.5 \%)$ \\
Vancouver Coastal Health Authorities) $_{\text {Caucasian }}{ }^{2}$ & $85(73.9 \%)$ \\
Past history of gastrointestinal issues & $44(38.3 \%)$ \\
Past history of allergic conditions & $100(87 \%)$ \\
Family history of Gl issues & $58(50.4 \%)$ \\
Family history of EoE & $7(6.1 \%)$ \\
Family history of allergic conditions & $92(80 \%)$ \\
\hline
\end{tabular}

${ }^{a}$ Non-Caucasians were predominantly South Asian
Table 2 Cross-sectional analysis

\begin{tabular}{|c|c|c|}
\hline Characteristic & $\begin{array}{l}\text { Median time } \\
\text { to diagnosis } \\
\text { in months (IQR) }\end{array}$ & $p$-value \\
\hline Sex & & 0.469 \\
\hline Male & $23(7,47.50)$ & \\
\hline Female & $19(6,36)$ & \\
\hline Location & & 0.623 \\
\hline Close to tertiary hospital & $21(9.50,46.50)$ & \\
\hline Far from tertiary hospital & $18.50(4,37.75)$ & \\
\hline Ethnicity & & $0.008^{*}$ \\
\hline Caucasian & $24(7,52)$ & \\
\hline Other & $12(4.50,23)$ & \\
\hline Past history of $\mathrm{Gl}$ issues & & 0.798 \\
\hline No & $23(4,49)$ & \\
\hline Yes & $18.50(7,38.75)$ & \\
\hline Past history of allergic conditions & & 0.917 \\
\hline No & $28(1,48)$ & \\
\hline Yes & $19(7,44.50)$ & \\
\hline Family history of $\mathrm{Gl}$ issues & & 0.937 \\
\hline No & $21(8,43)$ & \\
\hline Yes & $20(4,45.75)$ & \\
\hline Family history of EoE & & 0.977 \\
\hline Yes & $15(4,52)$ & \\
\hline No & $21(7,44.50)$ & \\
\hline Family history of allergic conditions & & 0.053 \\
\hline No & $12(1,29)$ & \\
\hline Yes & $23(9.25,47.50)$ & \\
\hline
\end{tabular}

explained $7.9 \%$ (Adjusted $\mathrm{R}^{2}=0.079$ ) of the total variance for our cohort.

This is the first study exploring TTD and possible predictors of TTD in a Canadian EoE cohort. Our finding of a TTD of 21 months (IQR $=7,45)$ is at the lower end of the range reported in a systematic review for pediatric patients (1.2-3.5 years) in North America and Europe, and much shorter than what has been found for adult EoE patients (3.0-8.0 years). [5] We were surprised that the TTD for our cohort was relatively short, and theorize that referring physicians being aware of our dedicated multidisciplinary clinic for $\mathrm{EoE}$ at $\mathrm{BCCH}$ may have contributed to decreased TTD through quicker referral times. As far as we know, no other EoE clinics in Canada exist where every patient is interviewed concurrently by a gastroenterologist and an allergist, with both specialists exchanging ideas together with the patient.

Our finding that Caucasians experience longer TTD could be partially explained by racial disparities in EoE presentation between Caucasians and other ethnicities such as South Asians, and suggests that differences in symptom presentation, as well as genetic and/or 
environmental factors, may influence TTD [6-8]. Given that South Asians are the second largest visible minority group in $\mathrm{BC}$, and the largest in Canada overall, we believe that our results are generalizable to the overall population of Canada, but may not be generalizable to individual provinces or territories with a different distribution of visible minority groups. [9]

Interestingly, older age at diagnosis predicted longer TTD $(p=0.006)$. This is in contrast to a study by Hruz et al. [10]. describing longer TTD in younger EoE patients. Their analysis, however, included mostly adult patients and used discrete age categories instead of a continuous age variable. We hypothesize that parents of older children may perceive their child's EoE symptoms as less worrisome than parents of younger children and may be less likely to seek medical attention for these symptoms, thereby increasing TTD. Additionally, older children may simply not tell their parents they are experiencing symptoms, or may engage in compensatory behaviours that help minimize their symptoms, which would also increase TTD. Older children may also be more likely to have other medical conditions to which they or their physician may attribute their EoE symptoms, which would increase TTD as well.

A study limitation was the use of patient self-report for certain independent variables. However, misclassification of these self-reported independent variables would have likely been non-differential, and hence should not have affected our results. In addition, our linear regression model explains only $7.9 \%$ of the overall variance, indicating that other factors not explored in our cohort have a significant effect on TTD.

In conclusion, our study is the first to explore the possible regional, genetic, and environmental factors that contribute to TTD for EoE patients in a pediatric and Canadian cohort. We found that Caucasians and children diagnosed at older ages experience greater TTD. Although our TTD is at the shorter end of the spectrum, further studies in other Canadian regions, more countries, and the use of objective measures, where possible, are needed to confirm our findings, and to determine strategies to increase EoE awareness and further shorten TTD.

\section{Abbreviations \\ EoE: Eosinophilic esophagitis; TTD: Time to diagnosis; BCCH: British Columbia Children's Hospital; BC: British Columbia; Gl: Gastrointestinal.}

\section{Acknowledgements}

We thank Christopher Mill and Timothy Teoh for initial assistance with the study, and Boris Kuzeljevic for providing statistical support.

\section{Authors' contributions}

ESC, LS, and JJ were involved in the design and conception of this study. EH, $V A, H K$, and JJ were involved in data acquisition. JJ and LS interpreted the data and contributed to writing the manuscript. All authors read and approved the final manuscript.

Funding

This study was funded by the British Columbia Children's Hospital Research Institute.

\section{Availability of data and materials}

Summary data and materials are available upon request.

\section{Ethics approval and consent to participate}

Ethics approval was obtained from the University of British Columbia Children's and Women's Research Ethics Board. Consent to participate was obtained from all study participants.

\section{Consent for publication}

Not applicable.

\section{Competing interests}

$\mathrm{JJ}, \mathrm{LS}$, and EH declare that they have no competing interests. VA has been an advisory board member at Avir Pharma. HK has received funding from Regeneron Pharmaceuticals and Adare Pharmaceuticals. ESC has received research support from DBV Technologies; has been a member of advisory boards for Pfizer, Pediapharm, Leo Pharma, Kaleo, DBV, AllerGenis; is a member of the healthcare advisory board for Food Allergy Canada; was an expert panel and coordinating committee member of the National Institute of Allergy and Infectious Diseases (NIAID)-sponsored Guidelines for Peanut Allergy Prevention; was co-lead of the CSACI oral immunotherapy guidelines, and was a member of the committee for the American Gastroenterological Association \& AAAAI/ACAAI Joint Task Force guidelines on the management of eosinophilic esophagitis.

\section{Author details}

${ }^{1}$ Faculty of Medicine, University of Toronto, Toronto, ON, Canada. ${ }^{2}$ BC Children's Hospital Research Institute, Vancouver, BC, Canada. ${ }^{3}$ Division of Allergy and Immunology, Department of Pediatrics, Faculty of Medicine, University of British Columbia, Vancouver, BC, Canada. ${ }^{4}$ Division

of Gastroenterology, Hepatology \& Nutrition, Department of Pediatrics, Faculty of Medicine, University of British Columbia, Vancouver, BC, Canada. ${ }^{5}$ Division of Gastroenterology, Faculty of Medicine, University of British Columbia, Vancouver, BC, Canada. ${ }^{6}$ St. Paul's Hospital, Vancouver, BC, Canada.

Received: 22 May 2020 Accepted: 5 October 2020

Published online: 14 October 2020

\section{References}

1. Carr S, Chan ES, Watson W. Correction to: Eosinophilic esophagitis. Allergy Asthma Clin Immun. 2019;15:22. https://doi.org/10.1186/s1322 3-019-0336-3.

2. Warners M, Oude Nijhuis R, de Wijkerslooth L, Smout A, Bredenoord A. The natural course of eosinophilic esophagitis and long-term consequences of undiagnosed disease in a large cohort. Am J Gastroenterol. 2018;113(6):836-44.

3. Cianferoni A, Spergel J. Eosinophilic esophagitis: a comprehensive review. Clinic Rev Allerg Immunol. 2016;50(2):159-74.

4. Harris P, Taylor R, Thielke R, Payne J, Gonzalez N, Conde J. Research electronic data capture (REDCap) - a metadata-driven methodology and workflow process for providing translational research informatics support. J Biomed Inform. 2009;42(2):377-81.

5. Shaheen NJ, Mukkada V, Eichinger CS, Schofield H, Todorova L, Falk GW. Natural history of eosinophilic esophagitis: a systematic review of epidemiology and disease course. Dis Esophagus. 2018;31:8. https://doi. org/10.1093/dote/doy015.

6. Ito J, Fujiwara T, Kojima R, Nomura I. Racial differences in eosinophilic gastrointestinal disorders among Caucasian and Asian. Allerology International. 2015;64(3):253-9. 
7. Weiler T, Mikhail I, Singal A, Sharma H. Racial differences in the clinical presentation of pediatric eosinophilic esophagitis. J Allergy Clin Immunol. 2014;2(3):320-5.

8. Teoh T, Koo C, Avinashi V, Chan ES. Characterization of ethnicity among children with eosinophilic esophagitis in British Columbia, Canada. J Allergy Clin Immunol. 2015;3(5):803-4.

9. Statistics Canada. https://www12.statcan.gc.ca/census-recensemen t/2016/dp-pd/hlt-fst/imm/Table.cfm?Lang=E\&T=44\&SP=1\&geo=59\&vi smin $=15 \& a g e=1 \&$ sex $=1$. Accessed 20 August 2020 .
10. Hruz P, Straumann A, Bussmann C, Heer P, Simon HU, Zwahlen M, Beglinger $C$, et al. Escalating incidence of eosinophilic esophagitis: A 20-year prospective, population-based study in Olten County, Switzerland. J Allergy Clin Immunol. 2011;128(6):1349-50.

\section{Publisher's Note}

Springer Nature remains neutral with regard to jurisdictional claims in published maps and institutional affiliations.
Ready to submit your research? Choose BMC and benefit from:

- fast, convenient online submission

- thorough peer review by experienced researchers in your field

- rapid publication on acceptance

- support for research data, including large and complex data types

- gold Open Access which fosters wider collaboration and increased citations

- maximum visibility for your research: over 100M website views per year

At BMC, research is always in progress.

Learn more biomedcentral.com/submissions 